\title{
Modified Crank-Nicolson Difference Schemes for Nonlocal Boundary Value Problem for the Schrödinger Equation
}

\author{
Allaberen Ashyralyev ${ }^{1}$ and Ali Sirma ${ }^{2}$ \\ ${ }^{1}$ Department of Mathematics, Fatih University, 34500 Büyükcekmece, Istanbul, Turkey \\ ${ }^{2}$ Department of Mathematics and Computer Sciences, Bahcesehir University, Besiktas, \\ 34353 Istanbul, Turkey \\ Correspondence should be addressed to Ali Sirma, ali.sirma@bahcesehir.edu.tr \\ Received 26 November 2008; Revised 30 March 2009; Accepted 19 June 2009 \\ Recommended by Leonid Berezansky
}

The nonlocal boundary value problem for Schrödinger equation in a Hilbert space is considered. The second-order of accuracy $r$-modified Crank-Nicolson difference schemes for the approximate solutions of this nonlocal boundary value problem are presented. The stability of these difference schemes is established. A numerical method is proposed for solving a one-dimensional nonlocal boundary value problem for the Schrödinger equation with Dirichlet boundary condition. A procedure of modified Gauss elimination method is used for solving these difference schemes. The method is illustrated by numerical examples.

Copyright (c) 2009 A. Ashyralyev and A. Sirma. This is an open access article distributed under the Creative Commons Attribution License, which permits unrestricted use, distribution, and reproduction in any medium, provided the original work is properly cited.

\section{Introduction}

In this article, the nonlocal boundary value problem for the Schrödinger equation

$$
\begin{gathered}
i u^{\prime}(t)+A u(t)=f(t), \quad 0<t<T, \\
u(0)=\sum_{m=1}^{p} \alpha_{m} u\left(\lambda_{m}\right)+\varphi, \\
0<\lambda_{1}<\lambda_{2}<\cdots<\lambda_{p} \leq T
\end{gathered}
$$

in a Hilbert space $H$ with the self-adjoint operator $A$ is considered. The Schrödinger equation plays an important role in the modeling of many phenomena. Methods of solutions for the 
Schrödinger equation have been studied extensively by many researchers (see, e.g., [1-9] and the references given therein).

The idea in this work is inspired from the works $[2,3,10,11]$. In the articles $[2,3]$ the existence and the uniqueness of the solution of the nonlocal boundary value problem (1.1) and its general form under some conditions are studied. In the article [8], to find an approximate solution of the problem (1.1), first-order of accuracy Rothe difference scheme and second-order of accuracy Crank-Nicolson difference scheme are presented. The stability estimates for the solution of this problem and the stability of these difference schemes are established.

The main aim of this paper is to study $r$ modified Crank-Nicolson difference schemes for the approximate solution of problem (1.1). The paper is organized as follows. In Section 2, we establish estimates for the stability of higher order derivatives of the solution of problem (1.1). In Section 3, the second-order of accuracy $r$ modified Crank-Nicolson difference schemes for the approximate solution of problem (1.1) are presented. The stabilities of these difference schemes are established. In Section 4, we study the convergence of these difference schemes. In Section 5, a numerical example is exposed in order to validate the schemes. A procedure involving the modified Gauss elimination method is used for solving these difference schemes.

Throughout this paper, the constants used are not necessarily the same at different occurrences.

\section{Nonlocal Boundary Value Problem}

Theorem 2.1. Assume that $f(t) \in C^{1}([0, T], H), \varphi \in D(A)$ and

$$
\sum_{m=1}^{p}\left|\alpha_{m}\right|<1
$$

Then there exists a unique solution $u(t)$ of problem (1.1) and the following inequalities are satisfied:

$$
\begin{aligned}
& \max _{0 \leq t \leq T}\|u(t)\|_{H} \leq C\left(\alpha_{1}, \ldots, \alpha_{p}\right)\left[\|\varphi\|_{H}+T \max _{0 \leq t \leq T}\|f(t)\|_{H}\right], \\
& \max _{0 \leq t \leq T}\left\|u^{\prime}(t)\right\|_{H}+\max _{0 \leq t \leq T}\|A u(t)\|_{H} \\
& \quad \leq C\left(\alpha_{1}, \ldots, \alpha_{p}\right)\left[\|A \varphi\|_{H}+T \max _{0 \leq t \leq T}\left\|f^{\prime}(t)\right\|_{H}+\|f(0)\|_{H}\right] .
\end{aligned}
$$

Proof. The proof of the estimate (2.2) is given in [8]. Now we will obtain the estimate (2.3). It is known that for smooth data of the problem

$$
i u^{\prime}(t)+A u(t)=f(t), \quad 0<t<T, \quad u(0)=\xi,
$$


there exists a unique solution of the problem (1.1), and the following formula holds:

$$
u(t)=e^{i A t} \xi-\int_{0}^{t} e^{i A(t-s)} i f(s) d s .
$$

Therefore we have

$$
A u(t)=e^{i A t} A \xi+f(0)+\int_{0}^{t} f^{\prime}(s) d s-f(0) e^{i A t}-\int_{0}^{t} e^{i A(t-s)} f^{\prime}(s) d s .
$$

So that we get the estimate

$$
\max _{0 \leq t \leq T}\|u(t)\|_{H} \leq\|A \xi\|_{H}+2\|f(0)\|_{H}+2 T \max _{0 \leq t \leq T}\left\|f^{\prime}(t)\right\|_{H}
$$

Using the condition $u(0)=\sum_{m=1}^{p} \alpha_{m} u\left(\lambda_{m}\right)+\varphi$ and the formula (2.6) we get

$$
\begin{aligned}
A \xi=R & \left\{\sum_{m=1}^{p} \alpha_{m} f(0)+\sum_{m=1}^{p} \alpha_{m} \int_{0}^{\lambda_{m}} f^{\prime}(s) d s-f(0) \sum_{m=1}^{p} \alpha_{m} e^{i A \lambda_{m}}\right. \\
& \left.-\sum_{m=1}^{p} \alpha_{m} \int_{0}^{\lambda_{m}} e^{i A\left(\lambda_{m}-s\right)} f^{\prime}(s) d s+A \varphi\right\}
\end{aligned}
$$

where

$$
R=\left(I-\sum_{m=1}^{p} \alpha_{m} e^{i A \lambda_{m}}\right)^{-1}
$$

By using estimates

$$
\|R\|_{H \rightarrow H} \leq \frac{1}{1-\sum_{m=1}^{p}\left|\alpha_{m}\right|} \leq C\left(\alpha_{1}, \ldots, \alpha_{p}\right), \quad\left\|e^{i A t}\right\|_{H \rightarrow H} \leq 1
$$


and the assumption $\sum_{m=1}^{p}\left|\alpha_{m}\right|<1$, we get

$$
\|A \xi\|_{H} \leq C\left(\alpha_{1}, \ldots, \alpha_{p}\right)\left\{2\|f(0)\|_{H}+2 T \max _{0 \leq t \leq T}\left\|f^{\prime}(t)\right\|_{H}+\|A \varphi\|_{H}\right\} .
$$

By using the estimates (2.7) and (2.11) we obtain an estimate for $A u$. Then by using the estimate for $A u$, the relation $i u^{\prime}(t)=f(t)-A u=f(0)+\int_{0}^{t} f^{\prime}(s) d s-A u$ and the triangle inequality we can obtain estimate (2.3). This completes the proof of Theorem 2.1.

\section{Difference Schemes, Stability}

In this section, we present $r$-modified Crank-Nicolson difference schemes for the approximate solutions of problem (1.1) and establish the stabilities of these difference schemes. It is assumed that $2 \tau \leq \lambda_{m}$ for $1 \leq m \leq p$. Let us associate the nonlocal boundary value problem (1.1) with the corresponding second-order of accuracy $r$-modified Crank-Nicolson difference schemes:

$$
\begin{gathered}
i \frac{u_{k}-u_{k-1}}{\tau}+\frac{A}{2}\left(u_{k}+u_{k-1}\right)=\varphi_{k}, \quad r+1 \leq k \leq N, \\
i \frac{u_{k}-u_{k-1}}{\tau}+A u_{k}=\varphi_{k}, \quad 1 \leq k \leq r, \\
u_{0}=\sum_{r \tau \geq \lambda_{m}} \alpha_{m}\left(\left(I+i l_{0 m} A\right) u_{l m}-i l_{0 m} \varphi_{l m}\right)+\sum_{\substack{r \tau<\lambda_{m} \\
\lambda_{m} / \tau \in Z^{+}}} \alpha_{m} u_{l m} \\
+\sum_{\substack{r \tau<\lambda_{m} \\
\lambda_{m} / \tau \notin Z^{+}}} \alpha_{m}\left(I+i d_{m} A\right) \frac{1}{2}\left(u_{l m}+u_{l m+1}\right)-i \sum_{\substack{r \tau<\lambda_{m} \\
\lambda_{m} / \tau \notin Z^{+}}} \alpha_{m} d_{m} \varphi_{l m}+\varphi, \\
0<\lambda_{1}<\lambda_{2}<\cdots<\lambda_{p} \leq T,
\end{gathered}
$$

for the approximate solutions of this nonlocal boundary value problem. $Z^{+}$denotes here the set $\{2, \ldots, n, \ldots\}$ and $l_{m}=\left\lfloor\lambda_{m} / \tau\right\rfloor, l_{0 m}=\lambda_{m}-\left\lfloor\lambda_{m} / \tau\right\rfloor \tau, d_{m}=\lambda_{m}-\left\lfloor\lambda_{m} / \tau\right\rfloor \tau-\tau / 2, \varphi_{k}=$ $f\left(t_{k}-\tau / 2\right), t_{k}=k \tau$, where $\lfloor x\rfloor$ stands for the greatest integer part of the real number $x$.

By [10],

$$
u_{k}= \begin{cases}R^{k} \xi-i \tau \sum_{j=1}^{k} R^{k-j+1} \varphi_{j}, & k=1, \ldots, r, \\ B^{k-r} R^{r} \xi-i \tau \sum_{j=1}^{r} B^{k-r} R^{r-j+1} \varphi_{j}-i \tau \sum_{j=r+1}^{k} B^{k-j} C \varphi_{j}, & k=r+1, \ldots, N\end{cases}
$$


is the solution of the $r$-modified Crank-Nicolson difference schemes for the approximate solutions of Cauchy problem

$$
\begin{gathered}
i \frac{u_{k}-u_{k-1}}{\tau}+\frac{A}{2}\left(u_{k}+u_{k-1}\right)=\varphi_{k}, \quad r+1 \leq k \leq N, \\
i \frac{u_{k}-u_{k-1}}{\tau}+A u_{k}=\varphi_{k}, \quad 1 \leq k \leq r, u_{0}=\xi .
\end{gathered}
$$

Here

$$
R=(I-i \tau A)^{-1}, \quad C=\left(I-i \frac{A}{2} \tau\right)^{-1}, \quad B=\left(I+i \frac{A}{2} \tau\right) C
$$

For $u_{0}$, using the formula (3.2) and the condition we obtain

$$
\begin{aligned}
\xi=T_{\tau}\{ & \left(-i \tau \sum_{r \tau \geq \lambda_{m}} \alpha_{m}\left(I+i l_{0 m} A\right) \sum_{j=1}^{l_{m}} R^{l_{m}-j+1} \varphi_{j}-i \sum_{r \tau \geq \lambda_{m}} \alpha_{m} l_{0 m} \varphi_{l m}\right) \\
& -i \tau \sum_{\substack{r \tau<\lambda_{m} \\
\lambda_{m} / \tau \in Z^{+}}} \alpha_{m}\left(\sum_{j=1}^{r} B^{l_{m}-r} R^{r-j+1} \varphi_{j}+\sum_{j=r+1}^{l_{m}} B^{l_{m}-j} C \varphi_{j}\right) \\
& -i \tau \sum_{\substack{r \tau<\lambda_{m} \\
\lambda_{m} / \tau \notin Z^{+}}} \alpha_{m}\left(I+i d_{m} A\right) \frac{1}{2}\left((I+B)\left(\sum_{j=1}^{r} B^{l_{m}-r} R^{r-j+1} \varphi_{j}+\sum_{j=r+1}^{l_{m}} B^{l_{m}-j} C \varphi_{j}\right)+C \varphi_{l_{m}+1}\right) \\
& \left.-i \sum_{\substack{r<<\lambda_{m} \\
\lambda_{m} / \tau \notin Z^{+}}} \alpha_{m} d_{m} A+\varphi\right\},
\end{aligned}
$$

where

$$
\begin{aligned}
T_{\tau}= & \left(I-\sum_{r \tau \geq \lambda_{m}} \alpha_{m}\left(I+i l_{0 m} A\right) R^{l_{m}}-\sum_{\substack{r \tau<\lambda_{m} \\
\lambda_{m} / \tau \in Z^{+}}} \alpha_{m} B^{l_{m}-r} R^{r}\right. \\
& -\sum_{\substack{r \tau<\lambda_{m} \\
\lambda_{m} / \tau \notin Z^{+}}} \alpha_{m}\left(I+i d_{m} A\right) \frac{1}{2}(I+B) B^{l_{m}-r} R^{r}
\end{aligned}
$$


Note that, here we considered $\sum_{k=r+1}^{l_{m}} B^{l_{m}-j} C \varphi_{j}=0$ for $l_{m}=r$. So, for the solution of problem (3.2), we have the following formula:

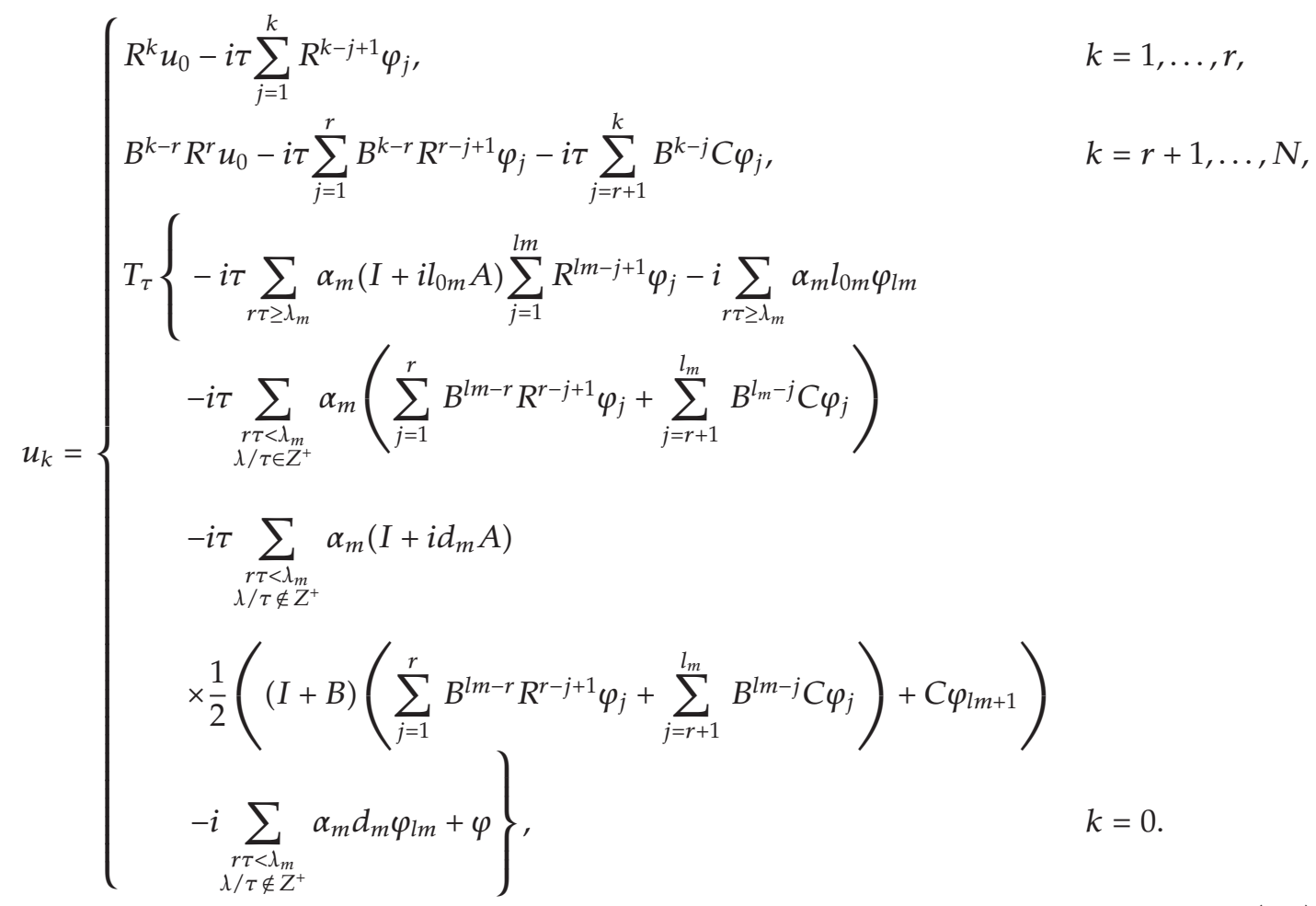

Theorem 3.1. Assume that $\varphi \in D(A)$ and

$$
\sum_{m=1}^{p}\left|\alpha_{m}\right|<1
$$

Then the solutions of the difference schemes (3.1) satisfy the stability inequalities

$$
\begin{aligned}
& \max _{0 \leq k \leq N}\left\|u_{k}\right\|_{H} \leq C\left(\alpha_{1}, \ldots, \alpha_{p}\right)\left[\|\varphi\|_{H}+T \max _{1 \leq k \leq N}\left\|\varphi_{k}\right\|_{H}\right], \\
& \max _{1 \leq k \leq N}\left\|\frac{u_{k}-u_{k-1}}{\tau}\right\|_{H}+\max _{1 \leq k \leq r}\left\|A u_{k}\right\|_{H}+\max _{r+1 \leq k \leq N}\left\|A \frac{u_{k}+u_{k-1}}{2}\right\|_{H} \\
& \quad \leq C\left(\alpha_{1}, \ldots, \alpha_{p}\right)\left[\|A \varphi\|_{H}+\left\|\varphi_{1}\right\|_{H} T \max _{2 \leq k \leq N}\left\|\frac{\varphi_{k}-\varphi_{k-1}}{\tau}\right\|_{H}\right] .
\end{aligned}
$$

Proof. Using the estimates

$$
\|R\|_{H \rightarrow H} \leq 1, \quad\|B\|_{H \rightarrow H} \leq 1, \quad\|C\|_{H \rightarrow H} \leq 1,
$$


and the formula (3.2), we can obtain

$$
\max _{1 \leq k \leq N}\left\|u_{k}\right\|_{H} \leq\left[\left\|u_{0}\right\|_{H}+T \max _{1 \leq k \leq N}\left\|\varphi_{k}\right\|_{H}\right]
$$

Using the spectral representation of the self-adjoint operators one can establish

$$
\left\|T_{\tau}\right\|_{H \rightarrow H} \leq \frac{1}{1-\sum_{m=1}^{p}\left|\alpha_{m}\right|} \leq C\left(\alpha_{1}, \ldots, \alpha_{p}\right)
$$

Estimate for $\left\|u_{0}\right\|_{H}$ should also be examined. By using formula (3.7), the triangle inequality, and estimates (3.11), (3.13) the following estimate is obtained:

$$
\left\|u_{0}\right\|_{H} \leq C\left(\alpha_{1}, \ldots, \alpha_{p}\right)\left[\|\varphi\|_{H}+2 T \max _{1 \leq k \leq N}\left\|\varphi_{k}\right\|_{H}\right] .
$$

The proof of the estimate (3.9) for the difference schemes (3.1) is based on the last estimate and estimate (3.12).

Now, estimate (3.10) will be obtained. Using (3.2), we get

$$
A u_{k}= \begin{cases}R^{k} A \xi-i \tau \sum_{j=1}^{k} A R^{k-j+1} \varphi_{j}, & k=1, \ldots, r, \\ B^{k-r} R^{r} A \xi-i \tau \sum_{j=1}^{r} B^{k-r} A R^{r-j+1} \varphi_{j}-i \tau \sum_{j=r+1}^{k} B^{k-j} A C \varphi_{j}, & k=r+1, \ldots, N .\end{cases}
$$

So that

$$
A u_{k}= \begin{cases}R^{k} A \xi+\left(\sum_{j=2}^{k} R^{k-j+1}\left(\varphi_{j-1}-\varphi_{j}\right)+\varphi_{k}-R^{k} \varphi_{1}\right), & k=1, \ldots, r, \\ B^{k-r} R^{r} A \xi+\sum_{j=2}^{r} B^{k-r} R^{r-j+1}\left(\varphi_{j-1}-\varphi_{j}\right)-B^{k-r} R^{r} \varphi_{1} & \\ \quad+\sum_{j=r+1}^{k} B^{k-j+1}\left(\varphi_{j-1}-\varphi_{j}\right)+\varphi_{k} & k=r+1, \ldots, N .\end{cases}
$$

For the estimate (3.10) the two cases should be examined separately: (i) $k=1, \ldots, r$, (ii) $k=r+1, \ldots, N$. Let $1 \leq k \leq r$. Then, using (3.16) we get

$$
\max _{1 \leq k \leq r}\left\|A u_{k}\right\|_{H} \leq\|R A \xi\|_{H}+2 N \max _{2 \leq k \leq N}\left\|\varphi_{k}-\varphi_{k-1}\right\|_{H}+2\left\|\varphi_{1}\right\|_{H}
$$

Therefore,

$$
\max _{1 \leq k \leq r}\left\|A u_{k}\right\|_{H} \leq\|R A \xi\|_{H}+2 T \max _{2 \leq k \leq N}\left\|\frac{\varphi_{k}-\varphi_{k-1}}{\tau}\right\|_{H}+2\left\|\varphi_{1}\right\|_{H}
$$


Estimate for $\|R A \xi\|_{H}$ should also be obtained. Using the formula (3.5) and the formula (3.16) we get

$$
\begin{aligned}
\xi=T_{\tau}\{ & \sum_{r \tau \geq \lambda_{m}} \alpha_{m}\left(I+i l_{0 m} A\right) R\left(\sum_{j=2}^{l_{m}} R^{l_{m}-j+1}\left(\varphi_{j-1}-\varphi_{j}\right)+\varphi_{l_{m}}-R^{l_{m}} \varphi_{1}\right) \\
& -i \sum_{r \tau \geq \lambda_{m}} \alpha_{m} l_{0 m} R A \varphi_{l_{m}}+\sum_{\substack{r \tau<\lambda_{m} \\
\lambda_{m} / \tau \in Z^{+}}} \alpha_{m} R\left(\sum_{j=2}^{r} B^{l_{m}-r} R^{r-j+1}\left(\varphi_{j-1}-\varphi_{j}\right)-R^{r} \varphi_{1}\right) \\
& +\sum_{\substack{r \tau<\lambda_{m} \\
\lambda_{m} / \tau \in Z^{+}}} \alpha_{m} R\left(\sum_{j=r+1}^{l_{m}} B^{l_{m}-j} C\left(\varphi_{j-1}-\varphi_{j}\right)+\varphi_{l_{m}}\right)+\sum_{\substack{r \tau<\lambda_{m} \\
\lambda_{m} / \tau \notin Z^{+}}} \alpha_{m}\left(I+i d_{m} A\right) \\
& \times \frac{1}{2} R\left(\sum_{j=2}^{r} B^{l_{m}-r} R^{r-j+1}\left(\varphi_{j-1}-\varphi_{j}\right)-R^{r} \varphi_{1}+\sum_{j=r+1}^{l_{m}} B^{l_{m}-j+1}\left(\varphi_{j-1}-\varphi_{j}\right)+\varphi_{l_{m}}\right) \\
& \left.+\left(\sum_{j=2}^{r} B^{l_{m}+1-r} R^{r-j+1}\left(\varphi_{j-1}-\varphi_{j}\right)-R^{r} \varphi_{1}+\sum_{j=r+1}^{l_{m}+1} B^{l_{m}-j+2}\left(\varphi_{j-1}-\varphi_{j}\right)+\varphi_{l_{m}+1}\right)\right) \\
& \left.-i \sum_{\substack{r \tau<\lambda_{m} \\
\lambda_{m} / \tau \notin Z^{+}}} \alpha_{m} d_{m} A R \varphi_{l_{m}}+R A \varphi\right\} .
\end{aligned}
$$

So that

$$
\|R A \xi\|_{H} \leq C_{1}\left(\alpha_{1}, \ldots, \alpha_{p}\right)\left[\|A \varphi\|_{H}+\left\|\varphi_{1}\right\|_{H}+T \max _{2 \leq k \leq N}\left\|\frac{\varphi_{k}-\varphi_{k-1}}{\tau}\right\|_{H}\right]
$$

Therefore, using the estimates (3.18) and (3.20) we obtain

$$
\max _{1 \leq k \leq r}\left\|A u_{k}\right\|_{H} \leq C_{2}\left(\alpha_{1}, \ldots, \alpha_{p}\right)\left[\|A \varphi\|_{H}+\left\|\varphi_{1}\right\|_{H}+T \max _{2 \leq k \leq N}\left\|\frac{\varphi_{k}-\varphi_{k-1}}{\tau}\right\|_{H}\right] .
$$

Then using the estimate for $A u_{k}$, the relation $i\left(\left(u_{k}-u_{k-1}\right) / \tau\right)=\varphi_{k}-A u_{k}=\varphi_{1}-\sum_{j=2}^{k}\left(\varphi_{j-1}-\right.$ $\left.\varphi_{j}\right)-A u_{k}$, and the triangle inequality we get the estimate

$$
\begin{aligned}
\max _{1 \leq k \leq r} & \left\|\frac{u_{k}-u_{k-1}}{\tau}\right\|_{H}+\max _{1 \leq k \leq r}\left\|A u_{k}\right\|_{H} \\
& \leq C_{2}\left(\alpha_{1}, \ldots, \alpha_{p}\right)\left[\|A \varphi\|_{H}+\left\|\varphi_{1}\right\|_{H}+T \max _{2 \leq k \leq N}\left\|\frac{\varphi_{k}-\varphi_{k-1}}{\tau}\right\|_{H}\right] .
\end{aligned}
$$


Now, let $k=r+1, \ldots, N$. Then using the formula (3.16) and the identity $(1 / 2)(I+B)=C$ we get

$$
\begin{aligned}
A \frac{u_{k}+u_{k-1}}{2}= & B^{k-r-1} C^{r-1} C R A \xi+\sum_{j=2}^{r} C B^{k-1-r} R^{r-j+1}\left(\varphi_{j-1}-\varphi_{j}\right)-R^{r} \varphi_{1} \\
& +\sum_{j=r+1}^{k} C B^{k-j}\left(\varphi_{j-1}-\varphi_{j}\right)+B\left(\varphi_{k-1}-\varphi_{k}\right)+\frac{\varphi_{k}+\varphi_{k-1}}{2} .
\end{aligned}
$$

So that

$$
\max _{r+1 \leq k \leq N}\left\|A \frac{u_{k}+u_{k-1}}{2}\right\|_{H} \leq\|R A \xi\|_{H}+3 T \max _{2 \leq k \leq N}\left\|\frac{\varphi_{k}-\varphi_{k-1}}{\tau}\right\|_{H}+3\left\|\varphi_{1}\right\|_{H} .
$$

Therefore, using the estimates (3.20) and (3.24), the estimate

$$
\max _{r+1 \leq k \leq N}\left\|A \frac{u_{k}+u_{k-1}}{2}\right\|_{H} \leq C_{3}\left(\alpha_{1}, \ldots, \alpha_{p}\right)\left[\|A \varphi\|_{H}+\left\|\varphi_{1}\right\|_{H}+T \max _{2 \leq k \leq N}\left\|\frac{\varphi_{k}-\varphi_{k-1}}{\tau}\right\|_{H}\right]
$$

is obtained. Then, by using the estimate (3.25), the relation $i\left(\left(u_{k}-u_{k-1}\right) / \tau\right)=\varphi_{k}-A\left(\left(u_{k}+\right.\right.$ $\left.\left.u_{k-1}\right) / 2\right)=\varphi_{1}-\sum_{j=2}^{k}\left(\varphi_{j-1}-\varphi_{j}\right)-A\left(\left(u_{k}+u_{k-1}\right) / 2\right)$, and the triangle inequality we get the estimate

$$
\begin{aligned}
& \max _{r+1 \leq k \leq N}\left\|\frac{u_{k}-u_{k-1}}{\tau}\right\|_{H}+\max _{r+1 \leq k \leq N}\left\|A \frac{u_{k}+u_{k-1}}{2}\right\|_{H} \\
& \quad \leq C_{4}\left(\alpha_{1}, \ldots, \alpha_{p}\right)\left[\|A \varphi\|_{H}+\left\|\varphi_{1}\right\|_{H}+T \max _{2 \leq k \leq N}\left\|\frac{\varphi_{k}-\varphi_{k-1}}{\tau}\right\|_{H}\right] .
\end{aligned}
$$

The result (3.10) follows from the estimates (3.22) and (3.26). So the proof is complete.

\section{Convergence}

Theorem 4.1. Assume that $\sum_{m=1}^{p}\left|\alpha_{m}\right|<1$. Assume also that $A u^{\prime \prime}(t)(0 \leq t \leq T)$ and $u^{\prime \prime \prime}(t)(0 \leq t \leq$ $T)$ are continuous, then the solution of the difference scheme (3.1) satisfies the convergence estimate

$$
\max _{0 \leq k \leq N}\left\|u_{k}-u\left(t_{k}\right)\right\|_{H} \leq M^{*}(r) \tau^{2}
$$

where $M^{*}(r)$ does not depend on $\tau$ but depends on $r$. 
Proof. If we subtract (1.1) from (3.1) we obtain

$$
\begin{gathered}
i \frac{z_{k}-z_{k-1}}{\tau}+\frac{A}{2}\left(z_{k}+z_{k-1}\right)=A_{k}, \quad r+1 \leq k \leq N, \\
i \frac{z_{k}-z_{k-1}}{\tau}+A z_{k}=A_{k}, \quad 1 \leq k \leq r, \\
z_{0}=\sum_{r \tau \geq \lambda_{m}} \alpha_{m}\left(\left(I+i l_{0 m} A\right) z_{l m}-i l_{0 m} A_{l m}\right)+\sum_{\substack{r \tau<\lambda_{m} \\
\lambda_{m} / \tau \in Z^{+}}} \alpha_{m} z_{l m} \\
+\sum_{\substack{r \tau<\lambda_{m} \\
\lambda_{m} / \tau \notin Z^{+}}} \alpha_{m}\left(I+i d_{m} A\right) \frac{1}{2}\left(z_{l m}+z_{l m+1}\right)-i \sum_{\substack{r \tau<\lambda_{m} \\
\lambda_{m} / \tau \notin Z^{+}}} \alpha_{m} d_{m} A_{l m}+A_{0}, \\
0<\lambda_{1}<\lambda_{2}<\cdots<\lambda_{p} \leq T,
\end{gathered}
$$

where $z_{k}=u_{k}-u\left(t_{k}\right)$ and $A_{k}$ is defined by the formula

$$
A_{k}= \begin{cases}i\left(\frac{d}{d t}\left(u\left(t_{k-1 / 2}\right)\right)-\frac{u\left(t_{k}\right)-u\left(t_{k-1}\right)}{\tau}\right)+A\left(u\left(t_{k-1 / 2}\right)-u\left(t_{k}\right)\right), & 1 \leq k \leq r, \\ i\left(\frac{d}{d t}\left(u\left(t_{k-1 / 2}\right)\right)-\frac{u\left(t_{k}\right)-u\left(t_{k-1}\right)}{\tau}\right)+A\left(u\left(t_{k-1 / 2}\right)-\frac{u\left(t_{k}\right)+u\left(t_{k-1}\right)}{2}\right), & r+1 \leq r \leq N, \\ \sum_{r \geq \lambda_{m}} \alpha_{m}\left(I+i l_{0 m} A\right) u\left(t_{l m}\right)+\sum_{\substack{r \tau<\lambda_{m} \\ \lambda_{m} / \tau \in Z^{+}}} \alpha_{m} u\left(t_{l m}\right) & \\ +\sum_{\substack{r \tau<\lambda_{m} \\ \lambda_{m} / \tau \notin Z^{+}}}\left(I+i d_{m} A\right) \frac{1}{2}\left(u\left(t_{l m}\right)+u\left(t_{l m+1}\right)\right)-\sum_{m=1}^{p} \alpha_{m} u\left(\lambda_{m}\right) & k=0 . \\ +i \sum_{r \tau \geq \lambda_{m}} \alpha_{m} l_{0 m}\left(A_{l m}-\varphi_{l m}\right)+i \sum_{\substack{r \tau<\lambda_{m} \\ \lambda_{m} / \tau \notin Z^{+}}} \alpha_{m} d_{m}\left(A_{l m}-\varphi_{l m}\right), & \end{cases}
$$

Then the difference problem (4.2) has a solution in the form (3.7), but instead of $u_{k}, \varphi_{k}, \varphi$ we take $z_{k}, A_{k}, A_{0}, k=1, \ldots, N$, respectively. Using the estimates

$$
\|R\|_{H \rightarrow H} \leq 1, \quad\|B\|_{H \rightarrow H} \leq 1, \quad\|C\|_{H \rightarrow H} \leq 1,
$$

and the formula obtained for the solution of (4.2), we can obtain

$$
\begin{aligned}
& \max _{1 \leq k \leq r}\left\|z_{k}\right\|_{H} \leq\left[\left\|z_{0}\right\|_{H}+r \tau \max _{1 \leq k \leq r}\left\|A_{k}\right\|_{H}\right], \\
& \max _{r+1 \leq k \leq r}\left\|z_{k}\right\|_{H} \leq\left[\left\|z_{0}\right\|_{H}+T \max _{1 \leq k \leq r}\left\|A_{k}\right\|_{H}\right] .
\end{aligned}
$$


By the estimate (3.14) we have

$$
\left\|z_{0}\right\|_{H} \leq C\left(\alpha_{1}, \ldots, \alpha_{p}\right)\left[\left\|A_{0}\right\|_{H}+2 T \max _{1 \leq k \leq N}\left\|A_{k}\right\|_{H}\right]
$$

Therefore, in order to obtain the inequality (4.1) we need estimates for $A_{k}$ for $0 \leq k \leq N$.

For $0 \leq k \leq N$, by the use of the triangle inequality, Taylor's formula, continuity of $A u^{\prime \prime}(t)(0 \leq t \leq T)$ and $u^{\prime \prime \prime}(t)(0 \leq t \leq T)$, the estimates

$$
\max _{1 \leq k \leq r}\left\|A_{k}\right\|_{H} \leq M_{1} \tau, \quad \max _{r+1 \leq k \leq N}\left\|A_{k}\right\|_{H} \leq M_{2} \tau^{2}, \quad\left\|A_{0}\right\|_{H} \leq M_{2} \tau^{2}
$$

are obtained. From the last estimates the result follows.

\section{Numerical Results}

In this section, the numerical experiments of the nonlocal boundary value problem

$$
\begin{gathered}
i \frac{\partial u(t, x)}{\partial t}-\left((x+1) u_{x}\right)_{x}=f(t, x), \quad 0<t, x<1, \\
u(0, x)=\frac{1}{3} u\left(\frac{1}{2}, x\right)+\varphi(x), \quad 0<x<1, \\
u(t, 0)=u(t, 1)=0, \quad 0<t<1, \\
f(t, x)=\left[\pi^{2} \sin \pi x-\pi \cos \pi x+\pi^{2}(x+1) \sin \pi x\right] \exp (-i t), \\
\varphi(x)=\left(1-\frac{1}{3} \exp \left(-\frac{i}{2}\right)\right)(\sin \pi x) .
\end{gathered}
$$

by using modified Crank-Nicolson difference scheme (3.1) are investigated. The exact solution of this problem is

$$
u(t, x)=(\sin \pi x) \exp (-i t)
$$

For the approximate solution of problem (5.1), the set $[0,1]_{\tau} \times[0,1]_{h}$ of a family of grid points depending on the small parameters $\tau$ and $h$

$$
[0,1]_{\tau} \times[0,1]_{h}=\left\{\left(t_{k}, x_{n}\right): t_{k}=k \tau, 1 \leq k \leq N-1, N \tau=1, x_{n}=n h, 1 \leq n \leq M-1, M h=1\right\}
$$

is defined. 
Applying the second-order of accuracy modified Crank-Nicolson difference schemes (3.1) we present following second-order of accuracy difference schemes for the approximate solutions of problem (5.1)

$$
\begin{gathered}
i \frac{u_{n}^{k}-u_{n}^{k-1}}{\tau}-\frac{1}{2}\left(\frac{u_{n+1}^{k}-u_{n-1}^{k}}{2 h}+\frac{u_{n+1}^{k-1}-u_{n-1}^{k-1}}{2 h}\right)-\frac{x_{n}+1}{2}\left(\frac{u_{n+1}^{k}-2 u_{n}^{k}+u_{n-1}^{k}}{h^{2}}+\frac{u_{n+1}^{k-1}-2 u_{n}^{k-1}+u_{n-1}^{k-1}}{h^{2}}\right) \\
=f\left(t_{k-1 / 2}, x_{n}\right), \quad r+1 \leq k \leq N-1,1 \leq n \leq M-1, \\
i \frac{u_{n}^{k}-u_{n}^{k-1}}{\tau}-\frac{u_{n+1}^{k}-u_{n-1}^{k}}{2 h}-\left(x_{n}+1\right) \frac{u_{n+1}^{k}-2 u_{n}^{k}+u_{n-1}^{k}}{h^{2}} \\
=f\left(t_{k}-\frac{\tau}{2}, x_{n}\right), \quad 1 \leq k \leq r, 1 \leq n \leq M-1, \\
t_{k-1 / 2}=\left(\begin{array}{c}
\left.k-\frac{1}{2}\right) \tau, \quad x_{n}=n h, 1 \leq k \leq N, 1 \leq n \leq M-1, \\
u_{n}^{0}=\frac{1}{3} u_{n}^{[1 / 2 \tau]}+\varphi\left(x_{n}\right), \quad 1 \leq n \leq M-1, \\
u_{0}^{k}=0, \quad u_{M}^{k}=0, \quad 0 \leq k \leq N .
\end{array}\right.
\end{gathered}
$$

So for each $r$, we have $(N+1) \times(N+1)$ system of linear equations which can be written in the matrix form as

$$
\begin{gathered}
A_{n} U_{n+1}+B_{n} U_{n}+C_{n} U_{n-1}=D_{n} \varphi_{n}, \quad 1 \leq n \leq M-1, \\
U_{0}=0, \quad U_{M}=0,
\end{gathered}
$$

where

$$
\varphi_{n}=\left[\begin{array}{c}
\varphi_{n}^{0} \\
\varphi_{n}^{1} \\
\cdots \\
\varphi_{n}^{N}
\end{array}\right]_{(N+1) \times 1}, \quad \varphi_{n}^{k}= \begin{cases}\left(1-\frac{1}{3} \exp \left(-\frac{i}{2}\right)\right)\left(\sin \pi x_{n}\right), & k=0, \\
f\left(t_{k-1 / 2}, x_{n}\right), & 1 \leq k \leq N,\end{cases}
$$




$$
\begin{aligned}
& A_{n}=\left[\begin{array}{ccccccccc}
0 & a_{n} & 0 & \cdots & 0 & 0 & 0 & 0 & 0 \\
0 & 0 & a_{n} & \cdots & 0 & 0 & 0 & 0 & 0 \\
0 & 0 & 0 & \cdots & 0 & 0 & 0 & 0 & 0 \\
\cdots & \cdots & \cdots & \cdots & \cdots & \cdots & \cdots & \cdots & \cdots \\
0 & 0 & 0 & 0 & e_{n} & e_{n} & 0 & 0 & 0 \\
\cdots & \cdots & \cdots & \cdots & \cdots & \cdots & \cdots & \cdots & \cdots \\
0 & 0 & 0 & \cdots & 0 & 0 & e_{n} & e_{n} & 0 \\
0 & 0 & 0 & \cdots & 0 & 0 & 0 & e_{n} & e_{n} \\
0 & 0 & 0 & \cdots & 0 & 0 & 0 & 0 & 0
\end{array}\right], \\
& B_{n}=\left[\begin{array}{ccccccccccc}
b_{n} & c_{n} & 0 & \cdots & 0 & \cdots & 0 & 0 & 0 & 0 & 0 \\
0 & b_{n} & c_{n} & \cdots & 0 & \cdots & 0 & 0 & 0 & 0 & 0 \\
0 & 0 & 0 & \cdots & \cdots & \cdots & 0 & 0 & 0 & 0 & 0 \\
\cdots & \cdots & \cdots & \cdots & \cdots & \cdots & \cdots & \cdots & \cdots & \cdots & \cdots \\
0 & 0 & 0 & \cdots & 0 & \cdots & v_{n} & s_{n} & 0 & 0 & 0 \\
\cdots & \cdots & \cdots & \cdots & \cdots & \cdots & \cdots & \cdots & \cdots & \cdots & \cdots \\
0 & 0 & 0 & \cdots & 0 & \cdots & 0 & 0 & v_{n} & s_{n} & 0 \\
0 & 0 & 0 & \cdots & 0 & \cdots & 0 & 0 & 0 & v_{n} & s_{n} \\
1 & 0 & 0 & \cdots & -\frac{1}{3} & \cdots & 0 & 0 & 0 & 0 & 0
\end{array}\right] \text {, } \\
& C_{n}=\left[\begin{array}{ccccccccc}
0 & d_{n} & 0 & \cdots & 0 & 0 & 0 & 0 & 0 \\
0 & 0 & d_{n} & \cdots & 0 & 0 & 0 & 0 & 0 \\
0 & 0 & 0 & \cdots & 0 & 0 & 0 & 0 & 0 \\
\cdots & \cdots & \cdots & \cdots & \cdots & \cdots & \cdots & \cdots & \cdots \\
0 & 0 & 0 & \cdots & g_{n} & g_{n} & 0 & 0 & 0 \\
\cdots & \cdots & \cdots & \cdots & \cdots & \cdots & \cdots & \cdots & \cdots \\
0 & 0 & 0 & \cdots & 0 & 0 & g_{n} & g_{n} & 0 \\
0 & 0 & 0 & \cdots & 0 & 0 & 0 & g_{n} & g_{n} \\
0 & 0 & 0 & \cdots & 0 & 0 & 0 & 0 & 0
\end{array}\right] \text {, } \\
& D=I_{N+1}((N+1) \times(N+1) \text { identity matrix }) \text {, } \\
& U_{s}=\left[\begin{array}{c}
U_{s}^{0} \\
U_{s}^{1} \\
\cdots \\
U_{s}^{N-1} \\
U_{s}^{N}
\end{array}\right], \quad s=n-1, n, n+1 .
\end{aligned}
$$


Table 1: Comparison of the errors for the approximate solution of problem (5.1).

\begin{tabular}{lcccc}
\hline Method & $N=M=20$ & $N=M=40$ & $N=M=80$ & $N=M=160$ \\
\hline One-modified Crank-Nicholson & 0.0137 & 0.0038 & 0.0010 & 0.00025 \\
Two-modified Crank-Nicholson & 0.0226 & 0.0071 & 0.0019 & 0.00048 \\
Three-modified Crank-Nicholson & 0.0272 & 0.0099 & 0.0028 & 0.00072 \\
\hline
\end{tabular}

In the above matrices entries are given as

$$
\begin{gathered}
a_{n}=-\frac{1}{2 h}-\frac{n h+1}{h^{2}}, \quad b_{n}=-\frac{i}{\tau}, \quad c_{n}=\frac{i}{\tau}+\frac{2(n h+1)}{h^{2}}, \quad d_{n}=\left(\frac{1}{2 h}-\frac{n h+1}{h^{2}}\right) \\
e_{n}=-\frac{1}{4 h}-\frac{n h+1}{2 h^{2}}, \quad v_{n}=\left(-\frac{i}{\tau}+\frac{n h+1}{h^{2}}\right), \quad s_{n}=\frac{i}{\tau}+\frac{n h+1}{h^{2}}, \quad g_{n}=\frac{1}{4 h}-\frac{n h+1}{2 h^{2}} .
\end{gathered}
$$

Thus, we have the second-order difference equation (5.5) with respect to $n$ with matrix coefficients. To solve this difference equation we have applied the same modified Gauss elimination method for the difference equation with respect to $n$ with matrix coefficients. Hence, we seek a solution of the matrix in the following form:

$$
U_{n}=\alpha_{n+1} U_{n+1}+\beta_{n+1}, \quad n=M-1, \ldots, 2,1,0,
$$

where $\alpha_{j}(j=1, \ldots, M)$ are $(N+1) \times(N+1)$ square matrices and $\beta_{j}(j=1, \ldots, M)$ are $(N+1) \times 1$ column matrices defined by

$$
\alpha_{n+1}=-\left(B_{n}+C_{n} \alpha_{n}\right)^{-1} A_{n}, \quad \beta_{n+1}=\left(B_{n}+C_{n} \alpha_{n}\right)^{-1}\left(D \varphi_{n}-C_{n} \beta_{n}\right), \quad n=1,2,3, \ldots, M-1 .
$$

Note that for obtaining $\alpha_{n+1}, \beta_{n+1}, n=1, \ldots, M-1$, first we need to find $\alpha_{1}, \beta_{1}$. As in [8], we take $\alpha_{1}$ is an identity matrix, $\beta_{1}$ is the zero column vector.

For their comparison, first the errors computed by

$$
E_{M}^{N}=\max _{1 \leq k \leq N-1}\left(\sum_{n=1}^{M-1}\left|u\left(t_{k}, x_{n}\right)-u_{n}^{k}\right|^{2} h\right)^{1 / 2}
$$

of the numerical solutions of problem (5.1) are recorded for different values of $N$ and $M$, where $u\left(t_{k}, x_{n}\right)$ represents the exact solution and $u_{n}^{k}$ represents the numerical solution at $\left(t_{k}, x_{n}\right)$. The results are shown in Table 1 for $N=M=20,40,80$, and 160, respectively.

Second, for their comparison, the relative errors are computed by

$$
\operatorname{rel} E_{N}^{M}=\max _{1 \leq k \leq N} \frac{E_{N}^{M}}{\left(\sum_{n=1}^{M}\left|u\left(t_{k}, x_{n}\right)\right|^{2} h\right)^{1 / 2}},
$$

and Table 2 is constructed for $N=M=20,40,80$, and 160, respectively. 
Table 2: Relative errors for the approximate solution of problem (5.1).

\begin{tabular}{lcccc}
\hline Method & $N=M=20$ & $N=M=40$ & $N=M=80$ & $N=M=160$ \\
\hline One-modified Crank-Nicholson & 0.0194 & 0.0054 & 0.0014 & 0.00035 \\
Two-modified Crank-Nicholson & 0.0320 & 0.0101 & 0.0027 & 0.00069 \\
Three-modified Crank-Nicholson & 0.0385 & 0.0141 & 0.0040 & 0.00100 \\
\hline
\end{tabular}

In the article [12] it can also be found, an example that Crank-Nicolson difference scheme is divergent but modified Crank-Nicolson is convergent.

\section{Acknowledgment}

The authors are grateful to Mr. Tarkan Aydın (Bahcesehir University, Turkey) for his comments and suggestions on implementation.

\section{References}

[1] M. E. Mayfield, Non-reflective boundary conditions for Schrödinger's equation, Ph.D. thesis, University of Rhode Island, Kingston, RI, USA, 1989.

[2] D. G. Gordeziani and G. A. Avalishvili, “Time-nonlocal problems for Schrödinger-type equations. I. Problems in abstract spaces," Differential Equations, vol. 41, no. 5, pp. 670-677, 2005.

[3] D. G. Gordeziani and G. A. Avalishvili, "Time-nonlocal problems for Schrödinger-type equations. II. Results for specific problems," Differential Equations, vol. 41, no. 6, pp. 813-819, 2005.

[4] H. Han, J. Jin, and X. Wu, "A finite-difference method for the one-dimensional time-dependent Schrödinger equation on unbounded domain," Computers E Mathematics with Applications, vol. 50, no. 8-9, pp. 1345-1362, 2005.

[5] J. Bourgain, "Growth of Sobolev norms in linear Schrödinger equations with quasi-periodic potential," Communications in Mathematical Physics, vol. 204, no. 1, pp. 207-247, 1999.

[6] X. Antoine, C. Besse, and V. Mouysset, "Numerical schemes for the simulation of the two-dimensional Schrödinger equation using non-reflecting boundary conditions," Mathematics of Computation, vol. 73, no. 248, pp. 1779-1799, 2004.

[7] A. Ashyralyev, S. Piskarev, and L. Weis, "On well-posedness of difference schemes for abstract parabolic equations in $L_{p}([0,1], E)$ spaces," Numerical Functional Analysis and Optimization, vol. 23, no. 7-8, pp. 669-693, 2002.

[8] A. Ashyralyev and A. Sirma, "Nonlocal boundary value problems for the Schrödinger equation," Computers \& Mathematics with Applications, vol. 55, no. 3, pp. 392-407, 2008.

[9] A. Ashyralyev and A. Sirma, "A note on the modified Crank-Nicolson difference schemes for Schrödinger equation," in Complex Analysis and Potential Theory (Proceedings of the Conference Satellite to ICM 2006), pp. 256-271, World Scientific Press, River Edge, NJ, USA, 2007.

[10] A. Ashyralyev, "Well-posedness of the modified Crank-Nicholson difference schemes in Bochner spaces," Discrete and Continuous Dynamical Systems. Series B, vol. 7, no. 1, pp. 29-51, 2007.

[11] P. E. Sobolevskii, Difference Methods for the Approximate Solution of Differential Equations, Izland. Voronezh. Gosud. Univ., Voronezh, Russia, 1975.

[12] A. Ashyralyev, A. S. Erdogan, and N. Arslan, "On the modified Crank-Nicholson difference schemes for parabolic equation with non-smooth data arising in biomechanics," Communications in Numerical Methods in Engineering. 


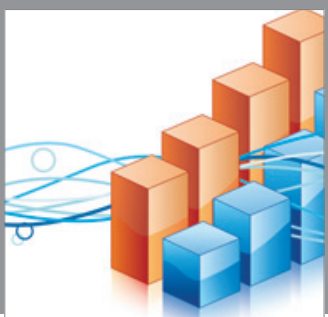

Advances in

Operations Research

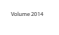

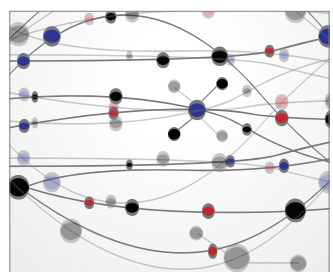

\section{The Scientific} World Journal
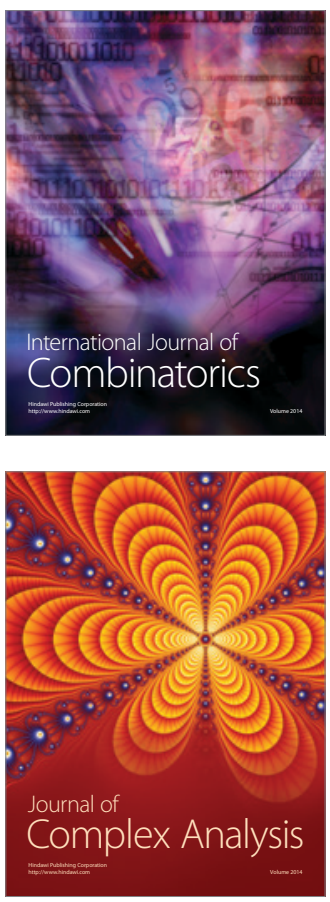

International Journal of

Mathematics and

Mathematical

Sciences
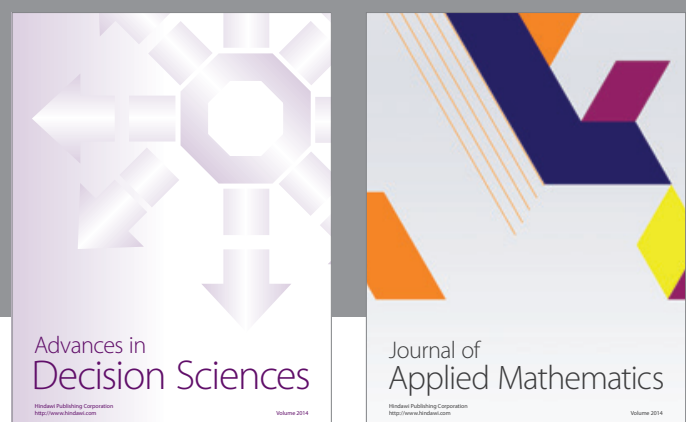

Journal of

Applied Mathematics
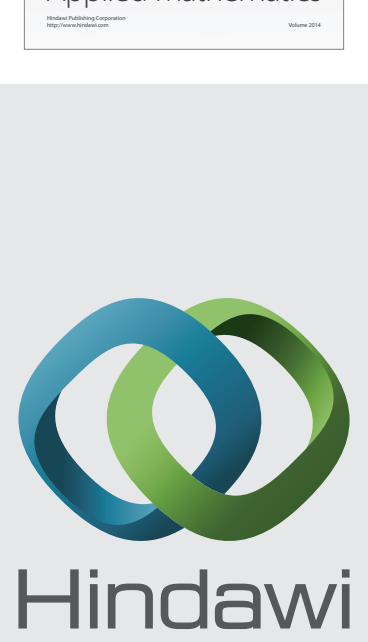

Submit your manuscripts at http://www.hindawi.com
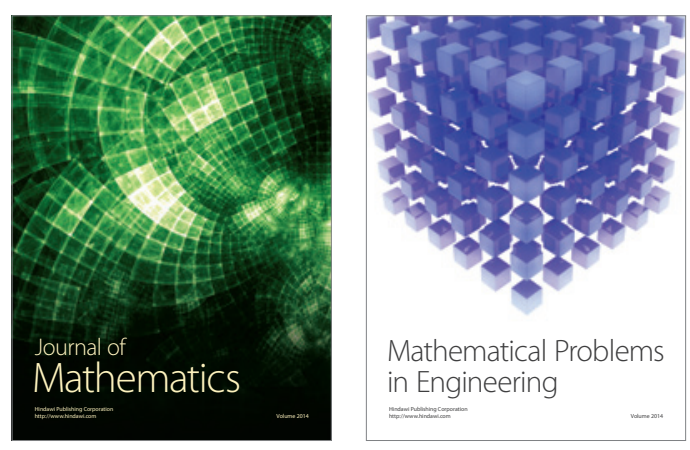

Mathematical Problems in Engineering
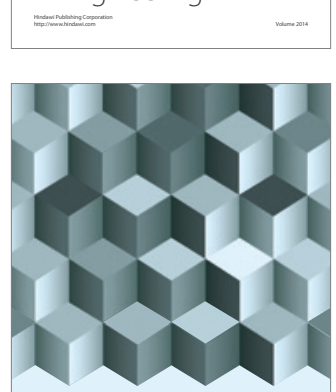

Journal of

Function Spaces
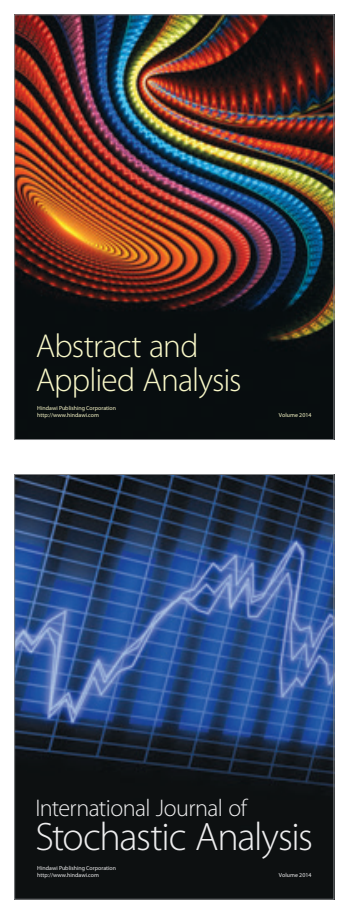

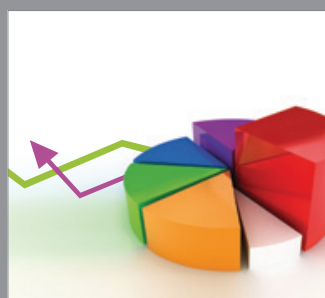

ournal of

Probability and Statistics

Promensencen
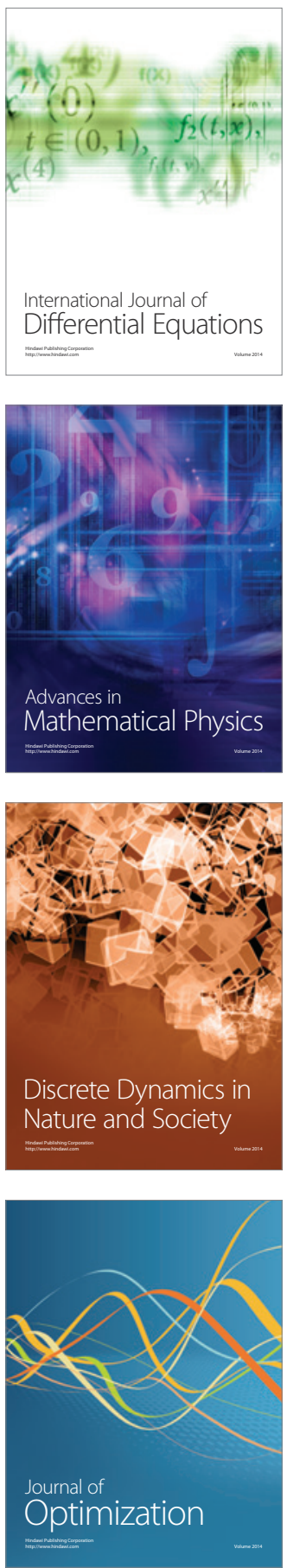